\title{
On Dualization in Products of Forests
}

\author{
Khaled M. Elbassioni` \\ Department of Computer Science, Rutgers University, 110 Frelinghuysen Road, \\ Piscataway NJ 08854-8003; (elbassio@paul.rutgers.edu).
}

\begin{abstract}
Let $\mathcal{P}=\mathcal{P}_{1} \times \ldots \times \mathcal{P}_{n}$ be the product of $n$ partially ordered sets, each with an acyclic precedence graph in which either the in-degree or the out-degree of each element is bounded. Given a subset $\mathcal{A} \subseteq \mathcal{P}$, it is shown that the set of maximal independent elements of $\mathcal{A}$ in $\mathcal{P}$ can be incrementally generated in quasi-polynomial time. We discuss some applications in data mining related to this dualization problem.
\end{abstract}

Keywords. Data mining, dualization, forest, incremental algorithms, poset.

\section{Introduction}

Given a finite set $V$ and a hypergraph $\mathcal{H} \subseteq 2^{V}$, the hypergraph dualization problem calls for enumerating all maximal independent sets of $\mathcal{H}$, i.e., all maximal subsets of $V$ that do not contain any hyperedge of $\mathcal{H}$. This problem has important applications in combinatorics [19], artificial intelligence [12], reliability theory [11, database theory [49], integer programming [79], and learning theory [5].

In this paper, we consider a natural generalization of this dualization problem which replaces edges of a hypergraph by a finite set of vectors over products of partially ordered sets (posets). Specifically, let $\mathcal{P} \stackrel{\text { def }}{=} \mathcal{P}_{1} \times \ldots \times \mathcal{P}_{n}$ be the product of $n$ posets. Let us use $\preceq$ to denote the precedence relation in $\mathcal{P}$ and also in $\mathcal{P}_{1}, \ldots, \mathcal{P}_{n}$, i.e., if $p=\left(p_{1}, \ldots, p_{n}\right) \in \mathcal{P}$ and $q=\left(q_{1}, \ldots, q_{n}\right) \in \mathcal{P}$, then $p \preceq q$ in $\mathcal{P}$ if and only if $p_{1} \preceq q_{1}$ in $\mathcal{P}_{1}, \ldots, p_{n} \preceq q_{n}$ in $\mathcal{P}_{n}$. For $\mathcal{A} \subseteq \mathcal{P}$, denote by $\mathcal{A}^{+}=\{x \in \mathcal{P} \mid x \succeq a$, for some $a \in \mathcal{A}\}$ and $2 \mathcal{A}^{-}=\{x \in \mathcal{P} \mid x \preceq$ $a$, for some $a \in \mathcal{A}\}$, the ideal and filter generated by $\mathcal{A}$. Any element in $\mathcal{P} \backslash \mathcal{A}^{+}$ is called independent of $\mathcal{A}$. Let $\mathcal{I}(\mathcal{A})$ be the set of all maximal independent elements for $\mathcal{A}$ (also referred to as the dual of $\mathcal{A}$ ):

$$
\mathcal{I}(\mathcal{A}) \stackrel{\text { def }}{=}\left\{p \in \mathcal{P} \mid p \notin \mathcal{A}^{+} \text {and }\left(q \in \mathcal{P}, q \succeq p, q \neq p \Rightarrow q \in \mathcal{A}^{+}\right)\right\} .
$$

Then for any $\mathcal{A} \subseteq \mathcal{P}$, we have the following decomposition of $\mathcal{P}$ :

$$
\mathcal{A}^{+} \cap \mathcal{I}(\mathcal{A})^{-}=\emptyset, \quad \mathcal{A}^{+} \cup \mathcal{I}(\mathcal{A})^{-}=\mathcal{P} .
$$

\footnotetext{
* Partially supported by DIMACS, the National Science Foundation's Center for Discrete Mathematics and Theoretical Computer Science.
} 
Given $\mathcal{A} \subseteq \mathcal{P}$, we consider the problem of incrementally generating all elements of $\mathcal{I}(\mathcal{A})$ :

$\operatorname{DUAL}(\mathcal{P}, \mathcal{A}, \mathcal{B}):$ Given a subset $\mathcal{A} \subseteq \mathcal{P}$ in a poset $\mathcal{P}$ and a collection of maximal independent elements $\mathcal{B} \subseteq \mathcal{I}(\mathcal{A})$, either find a new maximal independent element $p \in \mathcal{I}(\mathcal{A}) \backslash \mathcal{B}$, or prove that $\mathcal{A}$ and $\mathcal{B}$ form a dual pair: $\mathcal{B}=\mathcal{I}(\mathcal{A})$.

Clearly, the entire set $\mathcal{I}(\mathcal{A})$ can be generated by initializing $\mathcal{B}=\emptyset$ and iteratively solving the above problem $|\mathcal{I}(\mathcal{A})|+1$ times. If $\mathcal{P}$ is the Boolean cube, i.e., $\mathcal{P}_{i}=$ $\{0,1\}$ for all $i=1, \ldots, n$, we obtain the hypergraph dualization problem whose complexity is still an important open question. The best known algorithm runs in quasi-polynomial time $\operatorname{poly}(n, m)+m^{o(\log m)}$, where $m=|\mathcal{A}|+|\mathcal{B}|$, see 13 . For products of general partially ordered sets, it is not known whether the problem is NP-hard. In this note, it will be shown that the problem is unlikely to be NP-hard for products of posets whose precedence graphs are forests of (directed) trees with bounded degrees. Specifically, for $x \in \mathcal{P}_{i}$, denote by $x^{\perp}$ the set of immediate predecessors of $x$, i.e., $x^{\perp}=\left\{y \in \mathcal{P}_{i} \mid y \prec x, \quad \nexists z \in \mathcal{P}_{i}: y \prec z \prec x\right\}$, and let in- $\operatorname{deg}\left(\mathcal{P}_{i}\right)=\max \left\{\left|x^{\perp}\right|: x \in \mathcal{P}_{i}\right\}$. Similarly, denote by $x^{\top}$ the set of immediate successors of $x$, and let out-deg $\left(\mathcal{P}_{i}\right)=\max \left\{\left|x^{\top}\right|: x \in \mathcal{P}_{i}\right\}$. Throughout, let $m \stackrel{\text { def }}{=}|\mathcal{A}|+|\mathcal{B}|,[n] \stackrel{\text { def }}{=}\{1, \ldots, n\}, d \stackrel{\text { def }}{=} \max _{i \in[n]} \min \left\{\operatorname{in}-\operatorname{deg}\left(\mathcal{P}_{i}\right), \operatorname{out}-\operatorname{deg}\left(\mathcal{P}_{i}\right)\right\}$, and $\mu \stackrel{\text { def }}{=} \max \left\{\left|\mathcal{P}_{i}\right|: i \in[n]\right\}$. Our main result is the following:

Theorem 1. Problem $\operatorname{DUAL}(\mathcal{P}, \mathcal{A}, \mathcal{B})$ can be solved in poly $(n, m, \mu)+$ $m^{\text {d.o }(\log m)}$ time, if $\mathcal{P}$ is a product of forests.

In the next section, we discuss an application of Theorem 1 related to the generation of subsets of a poset that satisfy a certain monotone property. The proof of the theorem will be given in Sections 3, 4, 5, and 6.

\section{Generating Maximal Frequent and Minimal Infrequent Elements in a Database}

Let $\mathcal{P}=\mathcal{P}_{1} \times \ldots \times \mathcal{P}_{n}$ be the product of $n$ posets. Consider a database $\mathcal{D} \subseteq \mathcal{P}$ of transactions, each of which is an $n$-dimensional vector of attributes over $\mathcal{P}$. For an element $p \in \mathcal{P}$, let us denote by $S(p)=S_{\mathcal{D}}(p) \stackrel{\text { def }}{=}\{q \in \mathcal{D} \mid q \succeq p\}$, the set of transactions in $\mathcal{D}$ that support $p \in \mathcal{P}$. Note that, by this definition, the function $|S()|:. \mathcal{P} \mapsto \mathbb{Z}_{+}$is an anti-monotone function, i.e., $|S(p)| \leq|S(q)|$, whenever $p \succeq q$. Given $\mathcal{D} \subseteq \mathcal{P}$ and an integer threshold t, let us say that an element $p \in \mathcal{P}$ is $t$-frequent if it is supported by at least $t$ transactions in the database, i.e., if $\left|S_{\mathcal{D}}(p)\right| \geq t$. Conversely, $p \in \mathcal{P}$ is said to be $t$-infrequent if $\left|S_{\mathcal{D}}(p)\right|<t$. Since the function $\left|S_{\mathcal{D}}().\right|$ is anti-monotone, we may restrict our attention only to maximal frequent and minimal infrequent elements. Denote by $\mathcal{F}_{\mathcal{D}, t}$ the set of all minimal $t$-infrequent elements of $\mathcal{P}$ with respect to the database $\mathcal{D}$. Then $\mathcal{I}\left(\mathcal{F}_{\mathcal{D}, t}\right)$ is the set of all maximal $t$-frequent elements. Consider the following problem of incrementally generating all elements of $\mathcal{F}_{\mathcal{D}, t}$ : 
$\operatorname{GEN}\left(\mathcal{P}, \mathcal{F}_{\mathcal{D}, t}, \mathcal{X}\right):$ Given a subset $\mathcal{X} \subseteq \mathcal{F}_{\mathcal{D}, t} \subseteq \mathcal{P}$, either prove that $\mathcal{X}=\mathcal{F}_{\mathcal{D}, t}$ or find a new element in $\mathcal{F}_{\mathcal{D}, t} \backslash \mathcal{X}$.

The problem $\operatorname{GEN}\left(\mathcal{P}, \mathcal{I}\left(\mathcal{F}_{\mathcal{D}, t}\right), \mathcal{Y}\right)$ can be analogously defined. Although it might be hard in general to generate each of $\mathcal{F}_{\mathcal{D}, t}$ and $\mathcal{I}\left(\mathcal{F}_{\mathcal{D}, t}\right)$ separately (see [15 17]), the following joint generation problem may be easier:

$\operatorname{GEN}\left(\mathcal{P}, \mathcal{F}_{\mathcal{D}, t}, \mathcal{I}\left(\mathcal{F}_{\mathcal{D}, t}\right), \mathcal{X}, \mathcal{Y}\right):$ Given two explicitly listed collections $\mathcal{X} \subseteq \mathcal{F}_{\mathcal{D}, t}$ and $\mathcal{Y} \subseteq \mathcal{I}\left(\mathcal{F}_{\mathcal{D}, t}\right)$ in a poset $\mathcal{P}$, either find a new element in $\left(\mathcal{F}_{\mathcal{D}, t} \backslash \mathcal{X}\right) \cup\left(\mathcal{I}\left(\mathcal{F}_{\mathcal{D}, t}\right) \backslash\right.$ $\mathcal{Y})$, or prove that these collections are complete: $(\mathcal{X}, \mathcal{Y})=\left(\mathcal{F}_{\mathcal{D}, t}, \mathcal{I}\left(\mathcal{F}_{\mathcal{D}, t}\right)\right)$.

In fact, under the assumption that the precedence graph of each poset $\mathcal{P}_{i}$ is a rooted tree, Theorem 1, combined with the results of [6 8|15], implies that the two problems $\operatorname{GEN}\left(\mathcal{P}, \mathcal{F}_{\mathcal{D}, t}, \mathcal{I}\left(\mathcal{F}_{\mathcal{D}, t}\right), \mathcal{X}, \mathcal{Y}\right)$ and $\operatorname{GEN}\left(\mathcal{P}, \mathcal{F}_{\mathcal{D}, t}, \mathcal{X}\right)$ are unlikely to be NP-hard:

Theorem 2. If the precedence graph of each poset $\mathcal{P}_{i}$ is a forest in which the in-degree (or, alternatively, the out-degree) of each element is bounded by a constant, then problem $G E N\left(\mathcal{P}, \mathcal{F}_{\mathcal{D}, t}, \mathcal{I}\left(\mathcal{F}_{\mathcal{D}, t}\right), \mathcal{X}, \mathcal{Y}\right)$ is solvable in incremental quasi-polynomial time. If, further, each poset $\mathcal{P}_{i}$ is a join semi-lattice, (that $i s$, the precedence graph of $\mathcal{P}_{i}$ is a rooted tree for which $d=1$ ), then problem $\operatorname{GEN}\left(\mathcal{P}, \mathcal{F}_{\mathcal{D}, t}, \mathcal{X}\right)$ is also solvable in incremental quasi-polynomial time.

The separate and joint generation of maximal frequent and minimal infrequent elements of a poset are important tasks in knowledge discovery and data mining. If each poset $\mathcal{P}_{i}=\{0,1\}$, then these problems reduce to generating maximal frequent and minimal infrequent sets, which is used for mining association rules [1.214], correlations [10], sequential patterns [3], episodes [18], and many other applications. If the database $\mathcal{D}$ contains categorical (e.g., make of car), or quantitative (e.g., age, income) attributes, and the corresponding posets $\mathcal{P}_{i}$ are total orders, then the above generation problems can be used to mine the so called quantitative association rules [21. More generally, each attribute $a_{i}$ in the database can assume values belonging to some partially ordered set $\mathcal{P}_{i}$. For example, 20] describes applications where items in the database belong to sets of taxonomies (or is-a hierarchies), and proposes several algorithms for mining association rules among these hierarchical data (see also [16]). Note that, in this last example, each poset $\mathcal{P}_{i}$ has a tree precedence graph (in which all out-degrees are 1), and therefore, Theorem 2 applies.

\section{Preliminaries}

Let $\mathcal{P}=\mathcal{P}_{1} \times \ldots \times \mathcal{P}_{n}$, where the precedence graph of each poset $\mathcal{P}_{i}$ is a forest. Given two subsets $\mathcal{A} \subseteq \mathcal{P}$, and $\mathcal{B} \subseteq \mathcal{I}(\mathcal{A})$, we say that $\mathcal{B}$ is dual to $\mathcal{A}$ if $\mathcal{B}=\mathcal{I}(\mathcal{A})$, i.e., if $\mathcal{B}$ contains all the maximal elements of $\mathcal{P} \backslash \mathcal{A}^{+}$. Let us remark that, by (1), the latter condition is equivalent to $\mathcal{A}^{+} \cup \mathcal{B}^{-}=\mathcal{P}$.

Given any $\mathcal{Q} \subseteq \mathcal{P}$, let us denote by

$$
\mathcal{A}(\mathcal{Q})=\left\{a \in \mathcal{A} \mid a^{+} \cap \mathcal{Q} \neq \emptyset\right\}, \quad \mathcal{B}(\mathcal{Q})=\left\{b \in \mathcal{B} \mid b^{-} \cap \mathcal{Q} \neq \emptyset\right\},
$$


the subsets of $\mathcal{A}, \mathcal{B}$ whose ideal and filter respectively intersect $\mathcal{Q}$. A simple but an important observation, which will be used frequently in the algorithm below, is that

$$
\mathcal{Q} \subseteq \mathcal{A}^{+} \cup \mathcal{B}^{-} \Longleftrightarrow \mathcal{Q} \subseteq \mathcal{A}(\mathcal{Q})^{+} \cup \mathcal{B}(\mathcal{Q})^{-}
$$

Note that, for $a \in \mathcal{A}$ and $\mathcal{Q}=\mathcal{Q}_{1} \times \ldots \times \mathcal{Q}_{n}, a^{+} \cap \mathcal{Q} \neq \emptyset$ if and only if $a_{i}^{+} \cap \mathcal{Q}_{i} \neq \emptyset$, for all $i \in[n]$. Thus, the sets $\mathcal{A}(\mathcal{Q})$ and $\mathcal{B}(\mathcal{Q})$ can be found in $O(n m \mu)$ time.

To solve problem $D U A L(\mathcal{P}, \mathcal{A}, \mathcal{B})$, we shall use the same general approach used in 13 to solve the hypergraph dualization problem, by decomposing it into a number of smaller subproblems which are solved recursively. In each such subproblem, we start with a subposet $\mathcal{Q}=\mathcal{Q}_{1} \times \ldots \times \mathcal{Q}_{n} \subseteq \mathcal{P}$ (initially $\mathcal{Q}=\mathcal{P}$ ), and two subsets $\mathcal{A}(\mathcal{Q}) \subseteq \mathcal{A}$ and $\mathcal{B}(\mathcal{Q}) \subseteq \mathcal{B}$, and we want to check whether $\mathcal{A}(\mathcal{Q})$ and $\mathcal{B}(\mathcal{Q})$ are dual in $\mathcal{Q}$. As mentioned before, the latter condition is equivalent to checking whether $\mathcal{Q} \subseteq \mathcal{A}(\mathcal{Q})^{+} \cup \mathcal{B}(\mathcal{Q})^{-}$. To estimate the reduction in problem size from one level of the recursion to the next, we measure the change in the "volume" of the problem defined as $v=v(\mathcal{A}, \mathcal{B}) \stackrel{\text { def }}{=}|\mathcal{A}||\mathcal{B}|$. Since $\mathcal{B} \subseteq \mathcal{I}(\mathcal{A})$ is assumed, the following condition holds, by (11) for the original problem and all subsequent subproblems:

$$
a \npreceq b, \text { for all } a \in \mathcal{A}, b \in \mathcal{B} .
$$

Let $C(\mathcal{A}, \mathcal{B})=C(v(\mathcal{A}, \mathcal{B}))$ denote the number of subproblems that have to be solved in order to solve the original problem. We assume that $C(\mathcal{A}, \mathcal{B}) \leq$ $R(v(\mathcal{A}, \mathcal{B}))$ where $R(v)$ is a super-additive function of $v$ (i.e., $R(v)+R\left(v^{\prime}\right) \leq$ $R\left(v+v^{\prime}\right)$ for all $\left.v, v^{\prime} \geq 0\right)$.

We start with three propositions: Proposition 1 is useful for decomposing dualization on products of posets with disconnected precedence graphs into a number subproblems in which every poset has a connected precedence graph. Proposition 2 provides the base case for recursion. Proposition 3 states that a problem, closely related to the dualization problem, is NP-hard.

Proposition 1. Let $\mathcal{P}=\mathcal{P}_{1} \times \ldots \times \mathcal{P}_{n}$ and $\mathcal{A}, \mathcal{B} \subseteq \mathcal{P}$. Suppose that each poset $\mathcal{P}_{i}, i \in[n]$, can be partitioned into independent posets $\left(\mathcal{P}_{i}\right)_{1}, \ldots,\left(\mathcal{P}_{i}\right)_{k_{i}}$ (where two subposets $\mathcal{Q}$ and $\mathcal{R}$ are called independent if $q \npreceq r$ and $q \nsucceq r$ for all $q \in$ $\mathcal{Q}, r \in \mathcal{R})$. For every $y=\left(y_{1}, \ldots, y_{n}\right) \in\left[k_{1}\right] \times \ldots \times\left[k_{n}\right]$, if we let $\mathcal{P}^{y}=\left(\mathcal{P}_{1}\right)_{y_{1}} \times$ $\ldots \times\left(\mathcal{P}_{n}\right)_{y_{n}}, \mathcal{A}_{y}=\mathcal{A}\left(\mathcal{P}^{y}\right)$, and $\mathcal{B}_{y}=\mathcal{B}\left(\mathcal{P}^{y}\right)$, then

$$
\mathcal{P} \subseteq \mathcal{A}^{+} \cup \mathcal{B}^{-} \Longleftrightarrow \forall y \in\left[k_{1}\right] \times \ldots \times\left[k_{n}\right]: \mathcal{P}^{y} \subseteq \mathcal{A}_{y}^{+} \cup \mathcal{B}_{y}^{-} .
$$

Moreover, if for each $y \in\left[k_{1}\right] \times \ldots \times\left[k_{n}\right], C\left(\mathcal{A}_{y}, \mathcal{B}_{y}\right) \leq R\left(v\left(\mathcal{A}_{y}, \mathcal{B}_{y}\right)\right)$, then $C(\mathcal{A}, \mathcal{B}) \leq R(v(\mathcal{A}, \mathcal{B}))$.

Proposition 2. Suppose that $\min \{|\mathcal{A}|,|\mathcal{B}|\} \leq$ const, $\mathcal{A}, \mathcal{B} \subseteq \mathcal{P}$, then problem $\operatorname{DUAL}(\mathcal{P}, \mathcal{A}, \mathcal{B})$ is solvable in poly $(n, m, \mu)$ time.

Note that it is necessary to maintain the condition $\mathcal{A}, \mathcal{B} \subseteq \mathcal{P}$ in Proposition 2 Without this condition, the problem becomes NP-hard even for $\mathcal{B}=\emptyset$ : 
Proposition 3. Given a subposet $\mathcal{Q}$ of a poset $\mathcal{P}$ and a subset $\mathcal{A} \subseteq \mathcal{P}$, it is coNP-complete to decide if $\mathcal{Q} \subseteq \mathcal{A}^{+}$.

Clearly, $\mathcal{A}, \mathcal{B} \subseteq \mathcal{P}$ holds initially, but might not hold after decomposing $\mathcal{P}$. To solve this problem, we shall maintain the property that each poset $\mathcal{P}_{i}$ has a connected precedence graph. This will allow us to project the elements of $\mathcal{A}$ and $\mathcal{B}$ on the poset $\mathcal{P}$ without increasing their number. More precisely, if there is an $a \in \mathcal{A}, k \in[n]$ such that $a_{k}^{+} \cap \mathcal{P}_{k} \neq \emptyset$, but $a_{k} \notin \mathcal{P}_{k}$, we replace $a_{k}$ by $\min \left(a_{k}^{+} \cap \mathcal{P}_{k}\right) \stackrel{\text { def }}{=} \min \left\{x \in \mathcal{P}_{k} \mid x \succeq a_{k}\right\}$. Note that the existence of such a unique minimum is guaranteed by the fact that $\mathcal{P}_{k}$ has a connected precedence graph. Similarly, if there is a $b \in \mathcal{B}, k \in[n]$ such that $b_{k}^{-} \cap \mathcal{P}_{k} \neq \emptyset$, but $b_{k} \notin \mathcal{P}_{k}$, we replace $b_{k}$ by $\max \left(b_{k}^{-} \cap \mathcal{P}_{k}\right) \stackrel{\text { def }}{=} \max \left\{x \in \mathcal{P}_{k} \mid x \preceq b_{k}\right\}$. Note also that the duality condition (3) continues to hold after such replacements.

In the next section we develop several rules for decomposing a given dualization problem into smaller subproblems. The algorithm will select between these rules in such a way that the total volume is reduced from one iteration to the next.

\section{Decomposition Rules}

In general, the algorithm will decompose a given problem by selecting an $i \in[n]$ and partitioning $\mathcal{P}_{i}$ into two subposets $\mathcal{P}_{i}^{\prime}$ and $\mathcal{P}_{i}^{\prime \prime}$, defining accordingly two poset products $\mathcal{P}^{\prime}$ and $\mathcal{P}^{\prime \prime}$. Specifically, let $a^{o} \in \mathcal{A}, b^{o} \in \mathcal{B}$. By (3), there exists an $i \in[n]$, such that $a_{i}^{o} \npreceq b_{i}^{o}$. Let us assume, without loss of generality, that $i=1$ and set $\mathcal{P}_{1}^{\prime} \leftarrow \mathcal{P}_{1} \cap\left(a_{1}^{o}\right)^{+}, \mathcal{P}_{1}^{\prime \prime} \leftarrow \mathcal{P}_{1} \backslash \mathcal{P}_{1}^{\prime}$ (we may alternatively set $\mathcal{P}_{1}^{\prime \prime} \leftarrow \mathcal{P}_{1} \cap\left(b_{1}^{o}\right)^{-}$, and $\mathcal{P}_{1}^{\prime} \leftarrow \mathcal{P}_{1} \backslash \mathcal{P}_{1}^{\prime \prime}$, see Step 3 of the algorithm below). For brevity, we shall denote by $\overline{\mathcal{P}}$ the product $\mathcal{P}_{2} \times \ldots \times \mathcal{P}_{n}$, and accordingly by $\bar{q}$ the vector $\left(q_{2}, \ldots, q_{n}\right)$, for an element $q=\left(q_{1}, q_{2}, \ldots, q_{n}\right) \in \mathcal{P}$.

Defining $\mathcal{P}^{\prime}=\mathcal{P}_{1}^{\prime} \times \overline{\mathcal{P}}$ and $\mathcal{P}^{\prime \prime}=\mathcal{P}_{1}^{\prime \prime} \times \overline{\mathcal{P}}$ to be the two subposets induced by the above partitioning (see Fig. 1 - 1 a), and letting $\mathcal{A}^{\prime} \stackrel{\text { def }}{=} \mathcal{A}\left(\mathcal{P}^{\prime}\right)=\left\{a \in \mathcal{A} \mid a_{1} \succeq a_{1}^{o}\right\}$, $\mathcal{A}^{\prime \prime} \stackrel{\text { def }}{=} \mathcal{A} \backslash \mathcal{A}^{\prime}, \mathcal{B}^{\prime} \stackrel{\text { def }}{=} \mathcal{B}\left(\mathcal{P}^{\prime}\right)=\left\{b \in \mathcal{B} \mid b_{1} \succeq a_{1}^{o}\right\}, \mathcal{B}^{\prime \prime} \stackrel{\text { def }}{=} \mathcal{B} \backslash \mathcal{B}^{\prime}$, we conclude by (2) that $\mathcal{A}, \mathcal{B}$ are dual in $\mathcal{P}$ if and only if

$$
\begin{aligned}
& \mathcal{A}, \mathcal{B}^{\prime} \text { are dual in } \mathcal{P}^{\prime}, \quad \text { i.e., } \mathcal{P}^{\prime} \subseteq \mathcal{A}^{+} \cup\left(\mathcal{B}^{\prime}\right)^{-}, \text {and } \\
& \mathcal{A}^{\prime \prime}, \mathcal{B} \text { are dual in } \mathcal{P}^{\prime \prime}, \quad \text { i.e., } \mathcal{P}^{\prime \prime} \subseteq\left(\mathcal{A}^{\prime \prime}\right)^{+} \cup \mathcal{B}^{-} .
\end{aligned}
$$

As described above, it is required to maintain the property that each poset $\mathcal{P}_{i}$ has a connected precedence graph. Clearly, if $\mathcal{P}_{1}$ has a connected graph, then so does $\mathcal{P}_{1}^{\prime}$ by the above definitions (since $a_{1}^{o} \in \mathcal{P}_{1}^{\prime}$ ). However, this might not be the case for $\mathcal{P}_{1}^{\prime \prime}$, and thus let us denote the connected components of its precedence graph by $\left(\mathcal{P}_{1}^{\prime \prime}\right)_{1},\left(\mathcal{P}_{1}^{\prime \prime}\right)_{2} \ldots,\left(\mathcal{P}_{1}^{\prime \prime}\right)_{h}$. Let $\mathcal{A}_{j}^{\prime \prime} \stackrel{\text { def }}{=} \mathcal{A}^{\prime \prime}\left(\left(\mathcal{P}_{1}^{\prime \prime}\right)_{j} \times \overline{\mathcal{P}}\right)=\{a \in$ $\left.\mathcal{A}^{\prime \prime} \mid a_{1}^{+} \cap\left(\mathcal{P}_{1}^{\prime \prime}\right)_{j} \neq \emptyset\right\}$ and $\mathcal{B}_{j}^{\prime \prime} \stackrel{\text { def }}{=} \mathcal{B}^{\prime \prime}\left(\left(\mathcal{P}_{1}^{\prime \prime}\right)_{j} \times \overline{\mathcal{P}}\right)=\left\{b \in \mathcal{B}^{\prime \prime} \mid b_{1}^{-} \cap\left(\mathcal{P}_{1}^{\prime \prime}\right)_{j} \neq \emptyset\right\}$ 


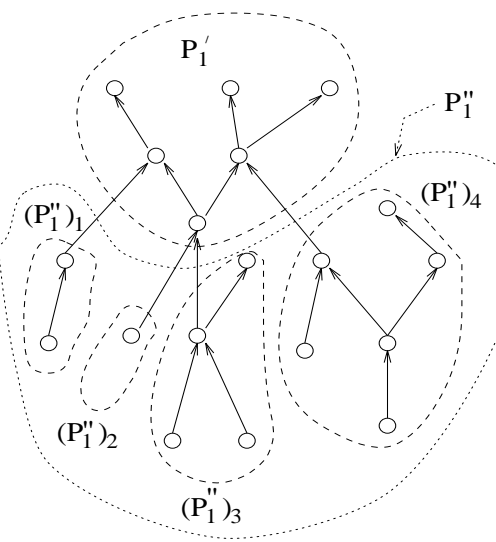

a: Rule (i).

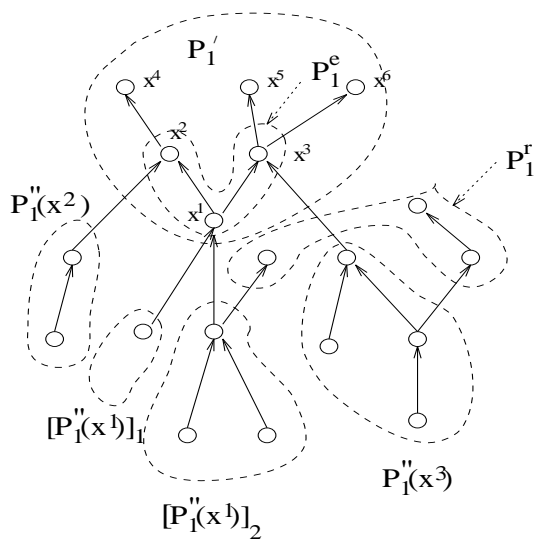

b: Rules (ii) and (iii).

Fig. 1. Decomposing the poset $\mathcal{P}_{1}$.

for $j=1, \ldots, h$. Then checking ([6) becomes equivalent to checking whether

$$
\left(\mathcal{P}_{1}^{\prime \prime}\right)_{j} \times \overline{\mathcal{P}} \subseteq\left(\mathcal{A}_{j}^{\prime \prime}\right)^{+} \cup\left(\mathcal{B}^{\prime} \cup \mathcal{B}_{j}^{\prime \prime}\right)^{-}, \quad j=1, \ldots, h .
$$

Thus we obtain our first decomposition rule:

Rule (i) Solve subproblem (5) together with $h$ subproblems (7).

Clearly, subproblems (5) and (6) are not independent. Once we know that (5) is satisfied, we gain some information about the solution of subproblem (6). To use this dependence, suppose that subproblem (15) has no solution, i.e., there exists no $q \in \mathcal{P} \backslash\left[\mathcal{A}^{+} \cup\left(\mathcal{B}^{\prime}\right)^{-}\right]$. Let us define $\mathcal{P}_{1}^{e}=\left\{x \in \mathcal{P}_{1}^{\prime} \mid x^{\perp} \cap \mathcal{P}_{1}^{\prime \prime} \neq \emptyset\right\}$ to be the set of elements in $\mathcal{P}_{1}^{\prime}$ with immediate predecessors in $\mathcal{P}_{1}^{\prime \prime}$ (see Fig. 1 $\mathrm{f}$ ). Let, for each $x \in \mathcal{P}_{1}^{e}$, the set $\mathcal{P}_{1}^{\prime \prime}(x)$ be the subtree of $x^{-}$, lying in $\mathcal{P}_{1}^{\prime \prime}$ and whose root in $\mathcal{P}_{1}^{\prime}$ is $x$, i.e., $\mathcal{P}_{1}^{\prime \prime}(x)=\left(x^{\perp} \cap \mathcal{P}_{1}^{\prime \prime}\right)^{-}$. Observe that $\mathcal{P}_{1}^{\prime \prime}(x)$ and $\mathcal{P}_{1}^{\prime \prime}(y)$ are independent posets for $x \neq y, x, y \in \mathcal{P}_{1}^{e}$ since the precedence graph of $\mathcal{P}_{1}$ is a forest. Let us further define, for each $x \in \mathcal{P}_{1}^{e}$, the sets

$$
\begin{aligned}
& \mathcal{A}^{\prime \prime}(x)=\mathcal{A}^{\prime \prime}\left(\mathcal{P}_{1}^{\prime \prime}(x) \times \overline{\mathcal{P}}\right), \widetilde{\mathcal{A}}(x)=\left\{a \in \mathcal{A} \mid a_{1} \preceq x\right\} \\
& \mathcal{B}^{\prime}(x)=\mathcal{B}^{\prime}\left(\mathcal{P}_{1}^{\prime \prime}(x) \times \overline{\mathcal{P}}\right), \quad \mathcal{B}^{\prime \prime}(x)=\mathcal{B}^{\prime \prime}\left(\mathcal{P}_{1}^{\prime \prime}(x) \times \overline{\mathcal{P}}\right)
\end{aligned}
$$

The following simple lemma is implied by the above definitions.

Lemma 1. For every $x \in \mathcal{P}_{1}^{e}$ and every $b \in \mathcal{B}^{\prime}(x)$, we must have $b_{1} \succeq x$.

Continuing, let $\mathcal{P}_{1}^{r}=\mathcal{P}_{1}^{\prime \prime} \backslash\left(\bigcup_{x \in \mathcal{P}_{1}^{e}} \mathcal{P}_{1}^{\prime \prime}(x)\right)$, let $\left(\mathcal{P}_{1}^{r}\right)_{1}, \ldots,\left(\mathcal{P}_{1}^{r}\right)_{k}$ be the connected components of $\mathcal{P}_{1}^{r}$, and let $\mathcal{A}_{j}^{r}=\mathcal{A}^{\prime \prime}\left(\left(\mathcal{P}_{1}^{r}\right)_{j} \times \overline{\mathcal{P}}\right), \mathcal{B}_{j}^{r}=\mathcal{B}^{\prime \prime}\left(\left(\mathcal{P}_{1}^{r}\right)^{j} \times \overline{\mathcal{P}}\right)$, for $j=1, \ldots, k$. We can now decompose subproblem (6) into

$$
\begin{aligned}
\mathcal{P}_{1}^{\prime \prime}(x) \times \overline{\mathcal{P}} \subseteq \mathcal{A}^{\prime \prime}(x)^{+} \cup\left(\mathcal{B}^{\prime \prime}(x) \cup \mathcal{B}^{\prime}(x)\right)^{-}, & x \in \mathcal{P}_{1}^{e}, \\
\left(\mathcal{P}_{1}^{r}\right)_{j} \times \overline{\mathcal{P}} \subseteq\left(\mathcal{A}_{j}^{r}\right)^{+} \cup\left(\mathcal{B}_{j}^{r}\right)^{-}, & j=1, \ldots, k .
\end{aligned}
$$


Given that (5) is satisfied, we claim that for each $x \in \mathcal{P}_{1}^{e}$, (8) is equivalent to

$$
\forall a \in \widetilde{\mathcal{A}}(x): \mathcal{P}_{1}^{\prime \prime}(x) \times\left(\overline{\mathcal{P}} \cap \bar{a}^{+}\right) \subseteq \mathcal{A}^{\prime \prime}(x)^{+} \cup \mathcal{B}^{\prime \prime}(x)^{-},
$$

where $\overline{\mathcal{P}} \cap \bar{a}^{+}=\left(\mathcal{P}_{2} \cap a_{2}^{+}\right) \times \ldots \times\left(\mathcal{P}_{n} \cap a_{n}^{+}\right)$. To see (10), we make use of the following:

Lemma 2. Given $z \in \mathcal{P}_{1}$, let $\mathcal{P}_{1}^{\prime}=\mathcal{P}_{1} \cap z^{+}, \mathcal{P}_{1}^{\prime \prime} \subseteq \mathcal{P}_{1} \cap z^{-} \backslash\{z\}$ be two disjoint subsets of $\mathcal{P}_{1}$. Define

$$
\begin{aligned}
& \mathcal{A}^{\prime \prime}=\left\{a \in \mathcal{A} \mid a_{1}^{+} \cap \mathcal{P}_{1}^{\prime \prime} \neq \emptyset\right\}, \quad \mathcal{A}^{\prime}=\left\{a \in \mathcal{A} \backslash \mathcal{A}^{\prime \prime} \mid a_{1}^{+} \cap \mathcal{P}_{1}^{\prime} \neq \emptyset\right\} \\
& \mathcal{B}^{\prime}=\left\{b \in \mathcal{B} \mid b_{1}^{-} \cap \mathcal{P}_{1}^{\prime} \neq \emptyset\right\}, \quad \mathcal{B}^{\prime \prime}=\left\{b \in \mathcal{B} \backslash \mathcal{B}^{\prime} \mid b_{1}^{-} \cap \mathcal{P}_{1}^{\prime \prime} \neq \emptyset\right\}
\end{aligned}
$$

Suppose further that we know that $\mathcal{P}_{1}^{\prime} \times \overline{\mathcal{P}} \subseteq\left(\mathcal{A}^{\prime} \cup \mathcal{A}^{\prime \prime}\right)^{+} \cup\left(\mathcal{B}^{\prime}\right)^{-}$, then

$$
\mathcal{P}_{1}^{\prime \prime} \times \overline{\mathcal{P}} \subseteq\left(\mathcal{A}^{\prime \prime}\right)^{+} \cup\left(\mathcal{B}^{\prime} \cup \mathcal{B}^{\prime \prime}\right)^{-} \Longleftrightarrow \forall a \in \widetilde{\mathcal{A}}: \mathcal{P}_{1}^{\prime \prime} \times\left(\overline{\mathcal{P}} \cap \bar{a}^{+}\right) \subseteq\left(\mathcal{A}^{\prime \prime}\right)^{+} \cup\left(\mathcal{B}^{\prime \prime}\right)^{-},
$$

where $\widetilde{\mathcal{A}}=\left\{a \in \mathcal{A}^{\prime} \cup \mathcal{A}^{\prime \prime} \mid a_{1} \preceq z\right\}$.

Proof. Suppose first that $\mathcal{P}_{1}^{\prime \prime} \times \overline{\mathcal{P}} \subseteq\left(\mathcal{A}^{\prime \prime}\right)+\cup\left(\mathcal{B}^{\prime} \cup \mathcal{B}^{\prime \prime}\right)^{-}$. Let $\left(q_{1}, \bar{q}\right) \in \mathcal{P}_{1}^{\prime \prime} \times\left(\overline{\mathcal{P}} \cap \bar{a}^{+}\right)$ for some $a \in \widetilde{\mathcal{A}}$, then $\left(q_{1}, \bar{q}\right) \in\left(\mathcal{A}^{\prime \prime}\right)^{+} \cup\left(\mathcal{B}^{\prime} \cup \mathcal{B}^{\prime \prime}\right)^{-}$. If $\left(q_{1}, \bar{q}\right) \preceq\left(b_{1}, \bar{b}\right) \in \mathcal{B}^{\prime}$, then by the definition of $\mathcal{B}^{\prime}$, there is a $y \in \mathcal{P}_{1}^{\prime}$ such that $y \preceq b_{1}$. But then, $a \in \widetilde{\mathcal{A}}, \bar{q} \in$ $\overline{\mathcal{P}} \cap \bar{a}^{+}$and $y \in \mathcal{P}_{1}^{\prime}$ imply that $\left(a_{1}, \bar{a}\right) \preceq(z, \bar{q}) \preceq(y, \bar{q}) \preceq\left(b_{1}, \bar{b}\right)$, which contradicts the assumed duality condition (3). This shows that $\left(q_{1}, \bar{q}\right) \in\left(\mathcal{A}^{\prime \prime}\right)+\cup\left(\mathcal{B}^{\prime \prime}\right)^{-}$.

For the other direction, let $\left(q_{1}, \bar{q}\right) \in\left(\mathcal{P}_{1}^{\prime \prime} \times \overline{\mathcal{P}}\right) \backslash\left(\mathcal{B}^{\prime}\right)^{-}$. Since $x \preceq y$ for all $x \in \mathcal{P}_{1}^{\prime \prime}, y \in \mathcal{P}_{1}^{\prime}$, we must have $(y, \bar{q}) \notin\left(\mathcal{B}^{\prime}\right)^{-}$for all $y \in \mathcal{P}_{1}^{\prime}$, for otherwise we get the contradiction $\left(q_{1}, \bar{q}\right) \preceq(y, \bar{q}) \preceq\left(b_{1}, \bar{b}\right)$ for some $b \in \mathcal{B}^{\prime}$. Now we use our assumption that $\mathcal{P}_{1}^{\prime} \times \overline{\mathcal{P}} \subseteq\left(\mathcal{A}^{\prime} \cup \mathcal{A}^{\prime \prime}\right)^{+} \cup\left(\mathcal{B}^{\prime}\right)^{-}$to conclude that $(y, \bar{q}) \in\left(\mathcal{A}^{\prime} \cup \mathcal{A}^{\prime \prime}\right)^{+}$ for all $y \in \mathcal{P}_{1}^{\prime}$. In particular, we have $(z, \bar{q}) \succeq\left(a_{1}, \bar{a}\right)$ for some $\left(a_{1}, \bar{a}\right) \in \mathcal{A}^{\prime} \cup \mathcal{A}^{\prime \prime}$. But this implies that $a \in \widetilde{\mathcal{A}}$ and hence that $\left(q_{1}, \bar{q}\right) \in \mathcal{P}_{1}^{\prime \prime} \times\left(\overline{\mathcal{P}} \cap \bar{a}^{+}\right)$for some $a \in \widetilde{\mathcal{A}}$. This gives $\left(q_{1}, \bar{q}\right) \in\left(\mathcal{A}^{\prime \prime}\right)^{+} \cup\left(\mathcal{B}^{\prime \prime}\right)^{-}$.

By considering the dual poset of $\mathcal{P}$ (that is, the poset $\mathcal{P}^{*}$ with the same set of elements as $\mathcal{P}$, but such that $x \prec y$ in $\mathcal{P}^{*}$ whenever $x \succ y$ in $\mathcal{P}$ ), and exchanging the roles of $\mathcal{A}$ and $\mathcal{B}$, we get the following symmetric version of Lemma 2 .

Lemma 3. Let $\mathcal{P}_{1}^{\prime \prime}=\mathcal{P}_{1} \cap z^{-}, \mathcal{P}_{1}^{\prime} \subseteq \mathcal{P}_{1} \cap z^{+} \backslash\{z\}$ be two disjoint subsets of $\mathcal{P}_{1}$ where $z \in \mathcal{P}_{1}$. Let $\mathcal{A}^{\prime \prime}, \mathcal{A}^{\prime}, \mathcal{B}^{\prime \prime}, \mathcal{B}^{\prime}$ be defined as in Lemma Q and let $\widetilde{\mathcal{B}}=\{b \in$ $\left.\mathcal{B}^{\prime} \cup \mathcal{B}^{\prime \prime} \mid b_{1} \succeq z\right\}$. Suppose we know that $\mathcal{P}_{1}^{\prime \prime} \times \overline{\mathcal{P}} \subseteq\left(\mathcal{A}^{\prime \prime}\right)^{+} \cup\left(\mathcal{B}^{\prime} \cup \mathcal{B}^{\prime \prime}\right)^{-}$, then

$$
\mathcal{P}_{1}^{\prime} \times \overline{\mathcal{P}} \subseteq\left(\mathcal{A}^{\prime} \cup \mathcal{A}^{\prime \prime}\right)^{+} \cup\left(\mathcal{B}^{\prime}\right)^{-} \Longleftrightarrow \forall b \in \widetilde{\mathcal{B}}: \mathcal{P}_{1}^{\prime} \times\left(\overline{\mathcal{P}} \cap \bar{b}^{-}\right) \subseteq\left(\mathcal{A}^{\prime}\right)^{+} \cup\left(\mathcal{B}^{\prime}\right)^{-} .
$$

Clearly, the equivalence of (8) and (10), given (5), is immediate from Lemma 2 by taking $z \leftarrow x$ and $\mathcal{P}_{1}^{\prime \prime} \leftarrow \mathcal{P}_{1}^{\prime \prime}(x)$. Then $\mathcal{P}_{1}^{\prime} \leftarrow x^{+} \cap \mathcal{P}_{1}, \mathcal{A}^{\prime \prime} \leftarrow \mathcal{A}^{\prime \prime}(x)$, $\mathcal{A}^{\prime} \leftarrow \mathcal{A}^{\prime}\left(\mathcal{P}_{1}^{\prime} \times \overline{\mathcal{P}}\right), \widetilde{\mathcal{A}} \leftarrow \widetilde{\mathcal{A}}(x), \mathcal{B}^{\prime \prime} \leftarrow \mathcal{B}^{\prime \prime}(x)$, and by Lemma $1 \mathcal{B}^{\prime} \leftarrow \mathcal{B}^{\prime}(x)$.

Now for each $x \in \mathcal{P}_{1}^{e}$, denoting by $\left[\mathcal{P}_{1}^{\prime \prime}(x)\right]_{1}, \ldots,\left[\mathcal{P}_{1}^{\prime \prime}(x)\right]_{k(x)}$ the connected components of $\mathcal{P}_{1}^{\prime \prime}(x)$, each problem of the form (10) can be further decomposed into the $k(x)$ subproblems:

$$
\left[\mathcal{P}_{1}^{\prime \prime}(x)\right]_{j} \times\left(\overline{\mathcal{P}} \cup \bar{a}^{+}\right) \subseteq \mathcal{A}_{j}^{\prime \prime}(x)^{+} \cup \mathcal{B}_{j}^{\prime \prime}(x)^{-}, \quad j=1, \ldots, k(x), \quad a \in \widetilde{\mathcal{A}}(x),
$$


where $\mathcal{A}_{j}^{\prime \prime}(x)=\left\{a \in \mathcal{A}^{\prime \prime}(x) \mid a_{1}^{+} \cap\left[\mathcal{P}_{1}^{\prime \prime}(x)\right]_{j} \neq \emptyset\right\}$, and $\mathcal{B}_{j}^{\prime \prime}(x)=\left\{b \in \mathcal{B}^{\prime \prime}(x) \mid b_{1}^{-} \cap\right.$ $\left.\left[\mathcal{P}_{1}^{\prime \prime}(x)\right]_{j} \neq \emptyset\right\}$. Thus we arrive at the following decomposition rule:

Rule (ii) Solve subproblem (5). If it has a solution then we get an element $q \in \mathcal{P} \backslash\left(\mathcal{A}^{+} \cup \mathcal{B}^{-}\right)$. Otherwise, we solve $|\widetilde{\mathcal{A}}(x)|$ subproblems (11), for each $x \in \mathcal{P}_{1}^{e}$ and $j=1, \ldots, k(x)$, and finally subproblems (9) for $j=1, \ldots, k$.

Suppose finally that subproblem (6), or its equivalent (7), has no solution (i.e. there is no $\left.q \in \mathcal{P}^{\prime \prime} \backslash\left[\left(\mathcal{A}^{\prime \prime}\right)+\cup \mathcal{B}^{-}\right]\right)$. We proceed in this case as follows. For $x \in \mathcal{P}_{1}$, let $\widetilde{\mathcal{A}}(x)=\left\{a \in \mathcal{A} \mid a_{1} \preceq x\right\}, \widetilde{\mathcal{B}}(x)=\left\{b \in \mathcal{B} \mid b_{1} \succeq x\right\}$, and $\hat{\mathcal{A}}^{\prime}(x)=\left\{a \in \mathcal{A}^{\prime} \mid a_{1}=x\right\}$. Let us use $x^{1}, \ldots, x^{w}$ to denote the elements of $\mathcal{P}_{1}^{\prime}$ and assume, without loss of generality, that they are topologically sorted in this order, that is, $x^{j} \prec x^{l}$ implies $j<l$ (see Fig. 1 b). Now we can use the following rule to solve our problem:

a. Solve subproblems (7), then

b. (decompose (5):) for $j=1$ to $w$, solve

$$
\left\{x^{j}\right\} \times \overline{\mathcal{P}} \subseteq\left[\left(\bigcup_{y \in\left(x^{j}\right)^{\perp}} \widetilde{\mathcal{A}}(y)\right) \cup \hat{\mathcal{A}}^{\prime}\left(x^{j}\right)\right]^{+} \cup \widetilde{\mathcal{B}}\left(x^{j}\right)^{-} .
$$

The following lemma will allow us to eliminate the contribution of the set $\mathcal{A}^{\prime \prime}$ in subproblems (12) at the expense of possibly introducing at most $|\mathcal{B}|^{d}$ additional subproblems.

Lemma 4. Given $x^{j} \in \mathcal{P}_{1}^{\prime}$, suppose we know that $\left(y^{-} \cap \mathcal{P}_{1}\right) \times \overline{\mathcal{P}} \subseteq \widetilde{\mathcal{A}}(y)^{+} \cup \mathcal{B}^{-}$ for all $y \in\left(x^{j}\right)^{\perp}$, then (12) is equivalent to

$$
\left\{x^{j}\right\} \times\left[\overline{\mathcal{P}} \cap\left(\bigcap_{y \in\left(x^{j}\right)^{\perp}} \bar{b}(y)^{-}\right)\right] \subseteq \hat{\mathcal{A}}^{\prime}\left(x^{j}\right)^{+} \cup \widetilde{\mathcal{B}}\left(x^{j}\right)^{-},
$$

for all collections $\left\{b(y) \in \widetilde{\mathcal{B}}(y) \mid y \in\left(x^{j}\right)^{\perp}\right\}$.

Proof. We prove by induction on $|Y|$, where $Y \subseteq\left(x^{j}\right)^{\perp}$, that

$$
\begin{aligned}
&\left\{x^{j}\right\} \times \overline{\mathcal{P}} \subseteq\left[\left(\bigcup_{y \in\left(x^{j}\right)^{\perp}} \widetilde{\mathcal{A}}(y)\right) \cup \hat{\mathcal{A}}^{\prime}\left(x^{j}\right)\right]^{+} \cup \widetilde{\mathcal{B}}\left(x^{j}\right)^{-} \Longleftrightarrow \\
&\left\{x^{j}\right\} \times\left[\overline{\mathcal{P}} \cap\left(\bigcap_{y \in Y} \bar{b}(y)^{-}\right)\right] \subseteq\left[\left(\bigcup_{y \in\left(x^{j}\right)^{\perp} \backslash Y} \widetilde{\mathcal{A}}(y)\right) \cup \hat{\mathcal{A}}^{\prime}\left(x^{j}\right)\right]^{+} \cup \widetilde{\mathcal{B}}\left(x^{j}\right)^{-},
\end{aligned}
$$

for all collections $\{b(y) \in \widetilde{\mathcal{B}}(y) \mid y \in Y\}$. This trivially holds for $Y=\emptyset$ and will prove the lemma for $Y=\left(x^{j}\right)^{\perp}$. To show (14), assume that it holds for some $Y \subset\left(x^{j}\right)^{\perp}$ and let $x \in\left(x^{j}\right)^{\perp} \backslash Y$. Consider a subproblem of the form

$$
\left\{x^{j}\right\} \times\left[\overline{\mathcal{P}} \cap\left(\bigcap_{y \in Y} \bar{b}(y)^{-}\right)\right] \subseteq\left[\widetilde{\mathcal{A}}(x) \cup\left(\bigcup_{y \in\left(x^{j}\right) \perp \backslash(Y \cup\{x\})} \widetilde{\mathcal{A}}(y)\right) \cup \hat{\mathcal{A}}^{\prime}\left(x^{j}\right)\right]^{+} \cup \widetilde{\mathcal{B}}\left(x^{j}\right)^{-},
$$


for some collection $\{b(y) \in \widetilde{\mathcal{B}}(y) \mid y \in Y\}$. Now we apply Lemma 3 with $z \leftarrow x$, $\mathcal{P}_{1}^{\prime \prime} \leftarrow x^{-} \cap \mathcal{P}_{1}, \mathcal{P}_{1}^{\prime} \leftarrow\left\{x^{j}\right\}, \mathcal{A}^{\prime \prime} \leftarrow \widetilde{\mathcal{A}}(x), \mathcal{A}^{\prime} \leftarrow\left(\bigcup_{y \in\left(x^{j}\right)^{\perp} \backslash(Y \cup\{x\})} \widetilde{\mathcal{A}}(y)\right) \cup \hat{\mathcal{A}}^{\prime}\left(x^{j}\right)$, and $\widetilde{\mathcal{B}} \leftarrow \widetilde{\mathcal{B}}(x)$ to get the required result.

Informally, Lemma 4 says that, given $x^{j} \in \mathcal{P}_{1}^{\prime}$, if the dualization subproblems for all sub-forests that lie below $x^{j}$ have been already verified to have no solution, then we can replace the solution to subproblem (12) by solving at most $\prod_{y \in\left(x^{j}\right)^{\perp}}|\widetilde{\mathcal{B}}(y)|$ subproblems of the form (13). Observe that it is important to check subproblems (12) in the topological order $j=1, \ldots, w$ to be able to use Lemma 4. Thus we get:

Rule (iii) Solve subproblems (7), and if they do not have a solution, then solve subproblems (13), for all collections $\left\{b(y) \in \widetilde{\mathcal{B}}(y) \mid y \in\left(x^{j}\right)^{\perp}\right\}$, for $j=1, \ldots, w$.

Finally it remains to remark that all the decomposition rules described above result, indeed, in posets with connected precedence graphs.

\section{The Algorithm}

Given subsets $\mathcal{A}, \mathcal{B} \subseteq \mathcal{P}=\mathcal{P}_{1} \times \ldots \times \mathcal{P}_{n}$ that satisfy the necessary duality condition (3), we proceed as follows:

Step 0. If the precedence graph of $\mathcal{P}_{i}$ is not connected, for some $i \in[n]$ (only can happen initially), use Proposition 1 to decompose the problem into a number of subproblems over posets with connected precedence graphs.

Step 1. For each $k \in[n]$ :

1. (eliminate:) if $a_{k}^{+} \cap \mathcal{P}_{k}=\emptyset$ for some $a \in \mathcal{A}\left(b_{k}^{-} \cap \mathcal{P}_{k}=\emptyset\right.$ for some $\left.b \in \mathcal{B}\right)$, then by (2), $a$ (respectively, $b$ ) can be discarded from further consideration;

2. (project:) if $a_{k} \prec \min \left(a_{k}^{+} \cap \mathcal{P}_{k}\right)$ for some $a \in \mathcal{A}\left(b_{k} \succ \max \left(b_{k}^{-} \cap \mathcal{P}_{k}\right)\right.$ for some $b \in \mathcal{B})$, then set $a_{k} \leftarrow \min \left(a_{k}^{+} \cap \mathcal{P}_{k}\right)$ (respectively, $b_{k} \leftarrow \max \left(b_{k}^{-} \cap \mathcal{P}_{k}\right)$ ).

Thus we may assume for next steps that $\mathcal{A}, \mathcal{B} \subseteq \mathcal{P}$.

Step 2. If $\min \{|\mathcal{A}|,|\mathcal{B}|\} \leq 3$, then the dualization problem can be solved in polynomial time using Proposition 2

Step 3. Let $a^{o}, b^{o}$ be arbitrary elements of $\mathcal{A}, \mathcal{B}$ respectively. Find an $i \in[n]$ such that $a_{i}^{o} \npreceq b_{i}^{o}$. Assume, without loss of generality, that $i=1$. If $\operatorname{in}-\operatorname{deg}\left(\mathcal{P}_{1}\right) \leq$ out-deg $\left(\mathcal{P}_{1}\right)$, we set $\mathcal{P}_{1}^{\prime} \leftarrow \mathcal{P}_{1} \cap\left(a_{1}^{o}\right)^{+}, \mathcal{P}_{1}^{\prime \prime} \leftarrow \mathcal{P}_{1} \backslash \mathcal{P}_{1}^{\prime}$, otherwise, we set $\mathcal{P}_{1}^{\prime \prime} \leftarrow$ $\mathcal{P}_{1} \cap\left(b_{1}^{o}\right)^{-}$, and $\mathcal{P}_{1}^{\prime} \leftarrow \mathcal{P}_{1} \backslash \mathcal{P}_{1}^{\prime \prime}$. In the latter case, we should use the symmetric versions of the decomposition rules (i)-(iii), listed above, which we obtain by exchanging the roles of $\mathcal{A}$ and $\mathcal{B}$ in these rules and replacing $\mathcal{P}$ by its dual poset $\mathcal{P}^{*}$. We assume therefore, without loss of generality, that the former case holds. Step 4 . Let $\mathcal{A}^{\prime}, \mathcal{A}^{\prime \prime}, \mathcal{B}^{\prime}, \mathcal{B}^{\prime \prime}, \ldots$ be as defined in the previous section, and define

$$
\epsilon^{\mathcal{A}}=\frac{\left|\mathcal{A}^{\prime}\right|}{|\mathcal{A}|}, \quad \epsilon^{\mathcal{B}}=\frac{\left|\mathcal{B}^{\prime \prime}\right|}{|\mathcal{B}|} .
$$

Observe that $\epsilon^{\mathcal{A}}>0$ and $\epsilon^{\mathcal{B}}>0$ since $a^{o} \in \mathcal{A}^{\prime}$ and $b^{o} \in \mathcal{B}^{\prime \prime}$. 
Step 5. Define

$$
\epsilon(v)=1 / \chi(v), \quad \text { where } \chi(v)^{\chi(v)}=v^{d}, v=v(\mathcal{A}, \mathcal{B}) .
$$

If $\min \left\{\epsilon^{\mathcal{A}}, \epsilon^{\mathcal{B}}\right\}>\epsilon(v)$, we use decomposition rule (i) which amounts to solving recursively subproblem (5) of volume $v\left(\mathcal{A}, \mathcal{B}^{\prime}\right)=|\mathcal{A}|\left|\mathcal{B}^{\prime}\right|$, and subproblems (7) of volumes $v\left(\mathcal{A}_{j}^{\prime \prime}, \mathcal{B}^{\prime} \cup \mathcal{B}_{j}^{\prime \prime}\right) \leq\left|\mathcal{A}_{j}^{\prime \prime}\right||\mathcal{B}|$, for $j=1, \ldots, h$. This gives rise to the recurrence

$$
C(v(\mathcal{A}, \mathcal{B})) \leq 1+C\left(|\mathcal{A}|\left|\mathcal{B}^{\prime}\right|\right)+\sum_{j=1}^{h} C\left(\left|\mathcal{A}_{j}^{\prime \prime}\right||\mathcal{B}|\right)
$$

Step 6 . If $\epsilon^{\mathcal{B}} \leq \epsilon(v)$, we apply rule (ii) and get the recurrence

$$
C(v) \leq 1+C\left(\left|\mathcal{A} \| \mathcal{B}^{\prime}\right|\right)+\sum_{x \in \mathcal{P}_{1}^{e}} \sum_{j=1}^{k(x)}|\widetilde{\mathcal{A}}(x)| C\left(\left|\mathcal{A}_{j}^{\prime \prime}(x)\right|\left|\mathcal{B}_{j}^{\prime \prime}(x)\right|\right)+\sum_{j=1}^{k} C\left(\left|\mathcal{A}_{j}^{r}\right|\left|\mathcal{B}_{j}^{r}\right|\right)
$$

Step 7. Finally, if $\epsilon^{\mathcal{A}} \leq \epsilon(v)<\epsilon^{\mathcal{B}}$, we use rule (iii) which gives

$$
C(v(\mathcal{A}, \mathcal{B})) \leq 1+\sum_{j=1}^{h} C\left(\left|\mathcal{A}_{j}^{\prime \prime}\right||\mathcal{B}|\right)+\sum_{j=1}^{w}\left(\prod_{y \in\left(x^{j}\right)^{\perp}}|\widetilde{\mathcal{B}}(y)|\right) C\left(\left|\hat{\mathcal{A}}^{\prime}\left(x^{j}\right)\right|\left|\widetilde{\mathcal{B}}\left(x^{j}\right)\right|\right) .
$$

\section{Proof of Theorem 1}

The proof essentially goes along the same lines as in 13 . We show by induction on $v=v(\mathcal{A}, \mathcal{B})$, that recurrences (15)-117) imply that $C(v) \leq R(v) \stackrel{\text { def }}{=} v^{\chi(v)}$. Since, for $\min \{|\mathcal{A}|,|\mathcal{B}|\} \leq 3$, Step 2 of the algorithm implies that $C(v)=1$, we may assume that $\min \{|\mathcal{A}|,|\mathcal{B}|\} \geq 4$, i.e., $v \geq 16$.

Let us consider first recurrence (15). Observe that $\mathcal{A}_{1}^{\prime \prime}, \ldots, \mathcal{A}_{h}^{\prime \prime}$ partition $\mathcal{A}^{\prime \prime}$ since $\mathcal{P}_{1}$ is a forest, and therefore, we get by the induction hypothesis and the super-additivity of $R($.

$$
\begin{aligned}
C(v) & \leq 1+R\left(|\mathcal{A}|\left|\mathcal{B}^{\prime}\right|\right)+\sum_{j=1}^{h} R\left(\left|\mathcal{A}_{j}^{\prime \prime}\right||\mathcal{B}|\right) \leq 1+R\left(|\mathcal{A}|\left|\mathcal{B}^{\prime}\right|\right)+R\left(\sum_{j=1}^{h}\left|\mathcal{A}_{j}^{\prime \prime} \| \mathcal{B}\right|\right) \\
& =1+R\left(\left(1-\epsilon^{\mathcal{B}}\right) v\right)+R\left(\left(1-\epsilon^{\mathcal{A}}\right) v\right) .
\end{aligned}
$$

Now using the facts that $\epsilon^{\mathcal{A}}>\epsilon(v), \epsilon^{\mathcal{B}}>\epsilon(v)$, and $v \geq 16$, we obtain by the monotonicity of $\mathcal{X}(v)$

$$
\begin{aligned}
C(v) & \leq 1+2[(1-\epsilon(v)) v]^{\chi((1-\epsilon(v)) v)} \leq 1+2[(1-\epsilon(v)) v]^{\chi(v)} \\
& =1+2\left(1-\frac{1}{\chi(v)}\right)^{\chi(v)} v^{\chi(v)} \leq 1+\frac{2}{e} v^{\chi(v)} \leq v^{\chi(v)}=R(v)
\end{aligned}
$$

concluding the induction proof for this case. 
Let us consider next (16) and observe that the sets $\mathcal{A}_{j}^{\prime \prime}(x), j=1, \ldots, k(x)$, $x \in \mathcal{P}_{1}^{e}$, are disjoint (again, since $\mathcal{P}_{1}$ is a forest), that $\mathcal{B}_{j}^{\prime \prime}(x) \subseteq \mathcal{B}^{\prime \prime}$ for all $x \in \mathcal{P}_{1}^{e}$ and $j=1, \ldots, k(x)$, and that the sets $\mathcal{B}_{j}^{r}, j=1, \ldots, k$ are disjoint and each is a subset of $\mathcal{B}^{\prime \prime}$. Consequently,

$$
\begin{aligned}
C(v) \leq 1 & +R\left(\left|\mathcal{A} \| \mathcal{B}^{\prime}\right|\right)+|\mathcal{A}| R\left(\sum_{x \in \mathcal{P}_{1}^{e}} \sum_{j=1}^{k(x)}\left|\mathcal{A}_{j}^{\prime \prime}(x)\right|\left|\mathcal{B}_{j}^{\prime \prime}(x)\right|\right) \\
& +R\left(\sum_{j=1}^{k}\left|\mathcal{A}_{j}^{r}\right|\left|\mathcal{B}_{j}^{r}\right|\right) \leq 1+R\left(\left(1-\epsilon^{\mathcal{B}}\right) v\right)+(|\mathcal{A}|+1) R\left(\epsilon^{\mathcal{B}} v\right) .
\end{aligned}
$$

Since $\min \{|\mathcal{A}|,|\mathcal{B}|\} \geq 4$ is assumed, we have $|\mathcal{A}|+1 \leq|\mathcal{A}||\mathcal{B}| / 3=v / 3$ and thus

$$
\begin{aligned}
C(v) & \leq 1+R\left(\left(1-\epsilon^{\mathcal{B}}\right) v\right)+\frac{v}{3} R\left(\epsilon^{\mathcal{B}} v\right) \leq R\left(\left(1-\epsilon^{\mathcal{B}}\right) v\right)+\frac{v}{2} R\left(\epsilon^{\mathcal{B}} v\right) \\
& \leq\left[\left(1-\epsilon^{\mathcal{B}}\right) v\right]^{\chi(v)}+\frac{v}{2}\left[\epsilon^{\mathcal{B}} v\right]^{\chi(v)}=\psi\left(\epsilon^{\mathcal{B}}\right) R(v),
\end{aligned}
$$

where $\psi(\epsilon) \stackrel{\text { def }}{=}(1-\epsilon)^{\chi(v)}+\frac{v}{2} \epsilon^{\chi(v)}$. Since $\psi(\epsilon)$ is convex in $\epsilon \in[0,1], \psi(0)=1$, $\epsilon^{\mathcal{B}} \leq \epsilon(v)$, and

$$
\psi(\epsilon(v))=\left(1-\frac{1}{\chi(v)}\right)^{\chi(v)}+\frac{v}{2}\left(\frac{1}{\chi(v)}\right)^{\chi(v)} \leq \frac{1}{e}+\frac{1}{2 v^{d-1}}<1,
$$

it follows that $\psi\left(\epsilon^{\mathcal{B}}\right)<1$ and hence, $C(v) \leq R(v)$.

Let us consider finally recurrence (17) and observe that $\left|\left(x^{j}\right)^{\perp}\right| \leq d$ for every $x^{j} \in \mathcal{P}_{1}^{\prime}$, by our assumption that in- $\operatorname{deg}\left(\mathcal{P}_{1}\right) \leq \operatorname{out}-\operatorname{deg}\left(\mathcal{P}_{1}\right)$ (see Step 3 of the algorithm). Thus (17) gives by induction

$$
C(v(\mathcal{A}, \mathcal{B})) \leq 1+\sum_{j=1}^{h} R\left(\left|\mathcal{A}_{j}^{\prime \prime}\right||\mathcal{B}|\right)+|\mathcal{B}|^{d} \sum_{j=1}^{w} R\left(\left|\hat{\mathcal{A}}^{\prime}\left(x^{j}\right)\right|\left|\widetilde{\mathcal{B}}\left(x^{j}\right)\right|\right) .
$$

Note that this the only place in which the bound $d$ on the degrees appears. Since the sets $\hat{\mathcal{A}}^{\prime}\left(x^{j}\right), j=1, \ldots, w$, partition $\mathcal{A}^{\prime}$, and $|\mathcal{B}|^{d} \leq v(|\mathcal{A}|,|\mathcal{B}|)^{d} / 3$ for $|\mathcal{A}| \geq 3$, we get $C(v) \leq 1+R\left(\left(1-\epsilon^{\mathcal{A}}\right) v\right)+\frac{v^{d}}{3} R\left(\epsilon^{\mathcal{A}} v\right)$. This implies by symmetry to 19 that $C(v) \leq R(v)$.

Note that $\chi(v)<\chi\left(m^{2}\right)<2 \chi(m) \sim 2 d \log m / \log \log m$, and we get the bound stated in Theorem 1 .

Acknowledgment. The author thanks Endre Boros, Vladimir Gurvich, and Leonid Khachiyan for many helpful discussions that led to this work.

\section{References}

1. R. Agrawal, T. Imielinski and A. Swami, Mining association rules between sets of items in massive databases. In Proc. the 1993 ACM-SIGMOD Int. Conf. Management of Data, pp. 207-216.

2. R. Agrawal and R. Srikant, Fast algorithms for mining association rules in large databases . In Proc. 20th Int. Conf. Very Large Data Bases, pp. 487-499, 1994. 
3. R. Agrawal and R. Srikant, Mining sequential patterns. In Proc. 11th Int. Conf. Data Engineering, pp. 3-14, 1995.

4. R. Agrawal, H. Mannila, R. Srikant, H. Toivonen and A. I. Verkamo, Fast discovery of association rules. In Advances in Knowledge Discovery and Data Mining, pp. 307-328, AAAI Press, Menlo Park, California, 1996.

5. M. Anthony and N. Biggs, Computational Learning Theory, Cambridge University Press, 1992.

6. J. C. Bioch and T. Ibaraki, Complexity of identification and dualization of positive Boolean functions, Information and Computation, 123 (1995) pp. 50-63.

7. E. Boros, K. Elbassioni, V. Gurvich, L. Khachiyan and K.Makino, On generating all minimal integer solutions for a monotone system of linear inequalities. In $A u$ tomata, Languages and Programming, 28th Int. Colloquium, ICALP 2001, LNCS 2076, pp. 92-103 (Springer Verlag, Berlin, Heidelberg, New York, July 2001).

8. E. Boros, V. Gurvich, L. Khachiyan and K. Makino, An inequality limiting the number of maximal frequent sets, DIMACS tech. report 2000-37, Rutgers Univ.

9. E. Boros, V. Gurvich, L. Khachiyan and K. Makino, Generating weighted transversals of a hypergraph, in Proceedings of the 2nd Japanese-Hungarian Symposium on Discrete Mathematics and Its Applications, pp. 13-22, Hungarian Academy of Sciences, Budapest, Hungary, April 20-23, 2001.

10. S. Brin, R. Motwani and C. Silverstein, Beyond market basket: Generalizing association rules to correlations. In Proc. the 1997 ACM-SIGMOD Int. Conf. Management of Data, pp. 265-276.

11. C. J. Colbourn, The combinatorics of network reliability, Oxford Univ.Press, 1987.

12. T. Eiter and G. Gottlob, Identifying the minimal transversals of a hypergraph and related problems, SIAM Journal on Computing, 24 (1995) pp. 1278-1304.

13. M. L. Fredman and L. Khachiyan, On the complexity of dualization of monotone disjunctive normal forms, Journal of Algorithms, 21 (1996) pp. 618-628.

14. D. Gunopulos, R. Khardon, H. Mannila, and H. Toivonen, Data mining, hypergraph transversals and machine learning. In Proc. 16th ACM-SIGACT-SIGMODSIGART Symposium on Principles of Database Systems, (1997) pp. 12-15.

15. V. Gurvich and L. Khachiyan, On generating the irredundant conjunctive and disjunctive normal forms of monotone Boolean functions, Discrete Applied Mathematics, 1996-97, issue 1-3, (1999) pp. 363-373.

16. J. Han and Y. Fu, Discovery of multiple-level association rules from large databases. In Proc. 21st Int. Conf. Very Large Data Bases, pp. 420-431, 1995.

17. K. Makino and T. Ibaraki, Interior and exterior functions of Boolean functions, Discrete Applied Mathematics, 69 (1996) pp. 209-231.

18. H. Mannila, and H. Toivonen, Discovery of frequent episodes in event sequences, Data Mining and Knowledge Discovery, 1 (1997) pp. 259-289.

19. R. C. Read, Every one a winner, or how to avoid isomorphism when cataloging combinatorial configurations, Annals of Discrete Math. 2 (1978) pp. 107-120.

20. R. Srikant and R. Agrawal, Mining generalized association rules. In Proc. 21st Int. Conf. Very Large Data Bases, pp. 407-419, 1995.

21. R. Srikant and R. Agrawal, Mining quantitative association rules in large relational tables. In Proc. of the ACM-SIGMOD 1996 Int. Conf. Management of Data, pp. 1-12, 1996. 